\title{
MS/MS-based networking and peptidogenomics guided genome mining revealed the stenothricin gene cluster in Streptomyces roseosporus
}

\author{
Wei-Ting Liu ${ }^{1}$, Anne Lamsa ${ }^{2}$, Weng Ruh Wong ${ }^{3}$, Paul D Boudreau ${ }^{4}$, Roland Kersten ${ }^{4}$, Yao Peng ${ }^{1}$, \\ Wilna J Moree ${ }^{5}$, Brendan M Duggan ${ }^{5}$, Bradley S Moore ${ }^{4}$, William H Gerwick ${ }^{4}$, Roger G Linington ${ }^{3}$, \\ Kit Pogliano ${ }^{2}$ and Pieter C Dorrestein ${ }^{1,4,5}$
}

\begin{abstract}
Most (75\%) of the anti-infectives that save countless lives and enormously improve quality of life originate from microbes found in nature. Herein, we described a global visualization of the detectable molecules produced from a single microorganism, which we define as the 'molecular network' of that organism, followed by studies to characterize the cellular effects of antibacterial molecules. We demonstrate that Streptomyces roseosporus produces at least four non-ribosomal peptide synthetase-derived molecular families and their gene subnetworks (daptomycin, arylomycin, napsamycin and stenothricin) were identified with different modes of action. A number of previously unreported analogs involving truncation, glycosylation, hydrolysis and biosynthetic intermediates and/or shunt products were also captured and visualized by creation of a map through MS/MS networking. The diversity of antibacterial compounds produced by $S$. roseosporus highlights the importance of developing new approaches to characterize the molecular capacity of an organism in a more global manner. This allows one to more deeply interrogate the biosynthetic capacities of microorganisms with the goal to streamline the discovery pipeline for biotechnological applications in agriculture and medicine. This is a contribution to a special issue to honor Chris Walsh's amazing career. The Journal of Antibiotics (2014) 67, 99-104; doi:10.1038/ja.2013.99; published online 23 October 2013
\end{abstract}

Keywords: BioMAP; biosynthesis; cyclic peptides; cytological profiling; mass spectrometry; metabolic exchange

\section{INTRODUCTION}

Since the discovery of penicillin in 1928 , scientists have been mining secreted microbial metabolites in the search for molecules to combat infectious diseases. ${ }^{1-3}$ Most (75\%) of the anti-infectives that save countless lives and enormously improve quality of life originate from microbes found in nature. ${ }^{3}$ Meanwhile, multidrug-resistant infections are increasing, and indeed deaths due to Staphylococcus aureus infections have overtaken deaths by HIV. ${ }^{4,5}$ The search for antimicrobials must continue with renewed vigor. ${ }^{6-9}$

Our laboratories have been interested in the development of mass spectrometric and genome mining methodologies with the goals of improving our functional understanding of these molecules and streamlining the therapeutic discovery process from natural sources. ${ }^{10-15}$ As opposed to studying one molecule at a time, we aim at a global visualization of molecules from a single microbe, which we define as the 'molecular network' of that organism, followed by studies to characterize the cellular effects of antibacterial molecules. In reality, it needs to be clear that the molecular network described and visualized using MS/MS networking is the detectable molecular network under the given mass spectrometric conditions. Here, the molecular network of Streptomyces roseosporus, a strain that due to its production of daptomycin is providing $600-700$ million dollars per year in revenue to CUBIST, is visualized and analyzed through MS/MS molecular networking, ${ }^{14}$ in combination with peptidogenomics (Figure 1). ${ }^{12,13}$ No other antibiotics were known to be produced by this strain before our laboratory started working with it and therefore would serve as a good proof-ofprinciple. MS/MS networking is a methodology wherein molecules that are characterized by mass spectrometry are subjected to fragmentation, and the resulting mass spectrometric patterns are aligned and cluster based on similarity. Each fragmentation is visualized as a node (circles), and the relatedness of each node is defined by an edge (lines). The thickness of the edge defines the degree of similarity of the MS/MS spectra. Molecular networking is a visualization tool that not only provides a global view of the molecular composition of a sample but also allows local interpretations, which can be used for applications such as elucidating biosynthesis or metabolic pathways, compare and

${ }^{1}$ Department of Chemistry and Biochemistry, University of California San Diego, La Jolla, CA, USA; ${ }^{2}$ Division of Biological Sciences, University of California San Diego, La Jolla, CA, USA: ${ }^{3}$ Department of Chemistry and Biochemistry, University of California Santa Cruz, Santa Cruz, CA, USA; ${ }^{4}$ Center for Marine Biotechnology and Biomedicine, University of California San Diego, La Jolla, CA, USA and ${ }^{5}$ Skaggs School of Pharmacy and Pharmaceutical Sciences, University of California San Diego, La Jolla, CA, USA Correspondence: Professor PC Dorrestein, Skaggs School of Pharmacy and Pharmaceutical Sciences, 9500 Gilman drive \# 0636, La Jolla, CA 92093, USA. E-mail: pdorrestein@ucsd.edu

Received 27 May 2013; revised 24 July 2013; accepted 27 July 2013; published online 23 October 2013 


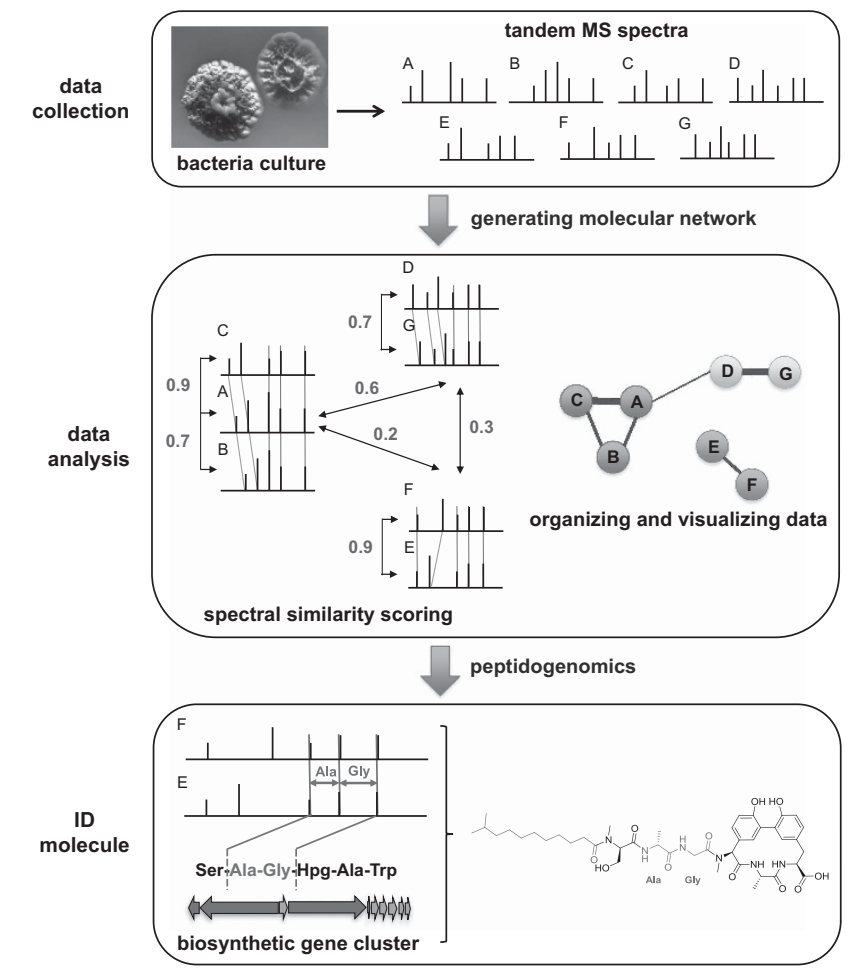

Figure 1 General workflow to characterize microbial metabolic profiles. Bacteria were cultured, and the tandem MS spectra of metabolites were collected. Spectra were aligned with the cosine scores calculating similarity with ' 1 ' indicating identical spectra and ' $O$ ' no similarity at all. A molecular network was thus generated with each node representing a unique spectrum and the thickness of edge representing the cosine score. The spectra that correlated to interesting nodes/subnetworks were subjected to sequence tagging and to query against the genome to identify biosynthetic gene subnetworks. By this process, the structures of molecules were predicted or identified with the information from both MS spectra and genomic information. A full color version of this figure is available at The Journal of Antibiotics journal online.

contrast hundreds, even thousands of data sets simultaneously, reveal biological responses to perturbations, facilitate therapeutic discoveries and much more. Although many applications can be envisioned with the ability to visualize the composition and relatedness of mass spectrometry detectable molecules in one network, here we use it to visualize structurally similar molecules from one organism. The data organization and visualization facilitate the mining of interesting molecular classes. Structurally divergent molecules that fragment in unique ways will form their own specific cluster or constellation, whereas related molecules cluster together. The molecules described in this paper are summarized in Supplementary Figure S1.

\section{RESULTS AND DISCUSSIONS}

To visualize the molecular network of $S$. roseosporus, extracts from a single Petri dish were subjected to direct infusion and LC-MS/MS in a data-dependent manner, as commonly used for untargeted metabolomics analysis. ${ }^{16,17}$ To reduce the complexity of analysis, MS/MS spectra with parent ion masses within $0.3 \mathrm{Da}$ and related MS/MS spectral patterns were merged, subjected to MS/MS networking ${ }^{14}$ and displayed in Cytoscape (Figure 2). ${ }^{18,19}$ The resulting networks contain 837 nodes covering a wide range of molecules that make up the molecular network of $S$. roseosporus (Figure 2). In this current work, we focus on a few constellations that are composed of subnetworks of
MS/MS spectra with peptidic signatures (Figure 2 and Supplementary Figure S2).

As $S$. roseosporus is known as a daptomycin producer, we first looked for daptomycin production and observed 15 nodes that are composed of the four known daptomycin variants and their corresponding natural isotopic species (Figure 2, Supplementary Figure S2). ${ }^{20-22}$ Unexpectedly, we also observed nodes in the daptomycin constellation that possess much lower molecular weights $(\mathrm{m} / \mathrm{z} 1280,1263)$ but clearly subnetworked with known daptomycin variants (Supplementary Figure S2). By comparing MS fragmentation patterns, the structures of these lower molecular weight analogs were identified as molecules that are structurally related to daptomycin but missing the $\mathrm{N}$-terminal lipid chain and tryptophan (Supplementary Figure S3).

We then looked for arylomycin, a molecular family produced by other Streptomyces strains that we recently discovered to be produced by S. roseosporus. ${ }^{13,23}$ All arylomycin variants formed their own MS/ MS subnetwork, which was composed of 12 nodes (Supplementary Figure S2). In addition, we observed arylomycin $\mathrm{Na}$ and $\mathrm{K}$ adducts located within a branch of the largest constellation (Supplementary Figures S4 and S5). Within this subnetwork there were ions $161 \mathrm{Da}$ smaller than the parent molecular family. Inspection of the MS/MS data revealed that the $-161 \mathrm{Da}$ component is at the C-terminal end of the arylomycin family and lacks the biaryl linkage as well as the C-terminal tryptophan residue previously seen in an ary $C$ mutant strain. ${ }^{24}$ It is intriguing that even in wild-type S. roseosporus, one can capture such biosynthetic intermediates.

The S. roseosporus molecular network contained two other constellations with peptidic signatures: one possessed 24 nodes and the other 141 nodes. Genome analysis suggested that S. roseosporus has the biosynthetic potential to produce napsamycins, but their production has not been documented. ${ }^{25}$ The napsamycins are a group of peptididyl nucleoside antibiotics, and herein we observed a potential napsamycin family as a constellation composed of 24 nodes (Figure 2, Supplementary Figure S2). The identity of these molecules as napsamycins was confirmed by comparing the MS/MS spectra with those previously reported (Supplementary Figure S6). ${ }^{25}$ Additionally, several higher molecular weight ions were found to subnetwork within the napsamycin family, and thus comprise as yet unknown napsamycin variants.

The constellation with 141 nodes possessed molecular ions ranging from $\mathrm{m} / \mathrm{z} 1090$ to 1473 and included the following series of fragment masses; $m / z$ 1090, 1104, 1118, 1132, 1146, 1160, 1174, 1188 (Supplementary Figure S2), suggesting a high likelihood that they were non-ribosomal peptide synthetase (NRPS)-derived. Therefore, we aligned the MS/MS spectra of these ions to reveal potential sequence tags, and matched these to the amino-acid building blocks that are predicted to be loaded by adenylation domains of the NRPS gene cluster found by antiSMASH (Figure 2). ${ }^{26}$ Of the seven NRPS gene subnetworks found in the $S$. roseosporus genome, four are short NRPS gene clusters containing less than four modules, and thus are less likely to be responsible for forming a peptide of mass near $1100 \mathrm{Da}$. Two of the remaining three larger NRPS gene clusters were assigned as the daptomycin and arylomycin gene clusters. ${ }^{13,22}$ MS/MS spectra of the unknown node revealed a potential sequence tag 151-83-87-86-83 (Supplementary Figure S7A). Because the $83 \mathrm{Da}$ mass could arise from dehydrobutyrine (derived from threonine), the sequence tag could be translated into X-Thr-Ser-X-Thr, which partially matched the putative sequence of the third gene cluster (Asp-Thr-Ser-Dab-Thr-Thr-Ser-Met-Gly) predicted by the Stachel-

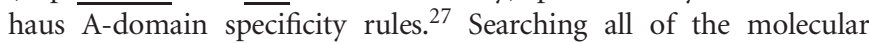




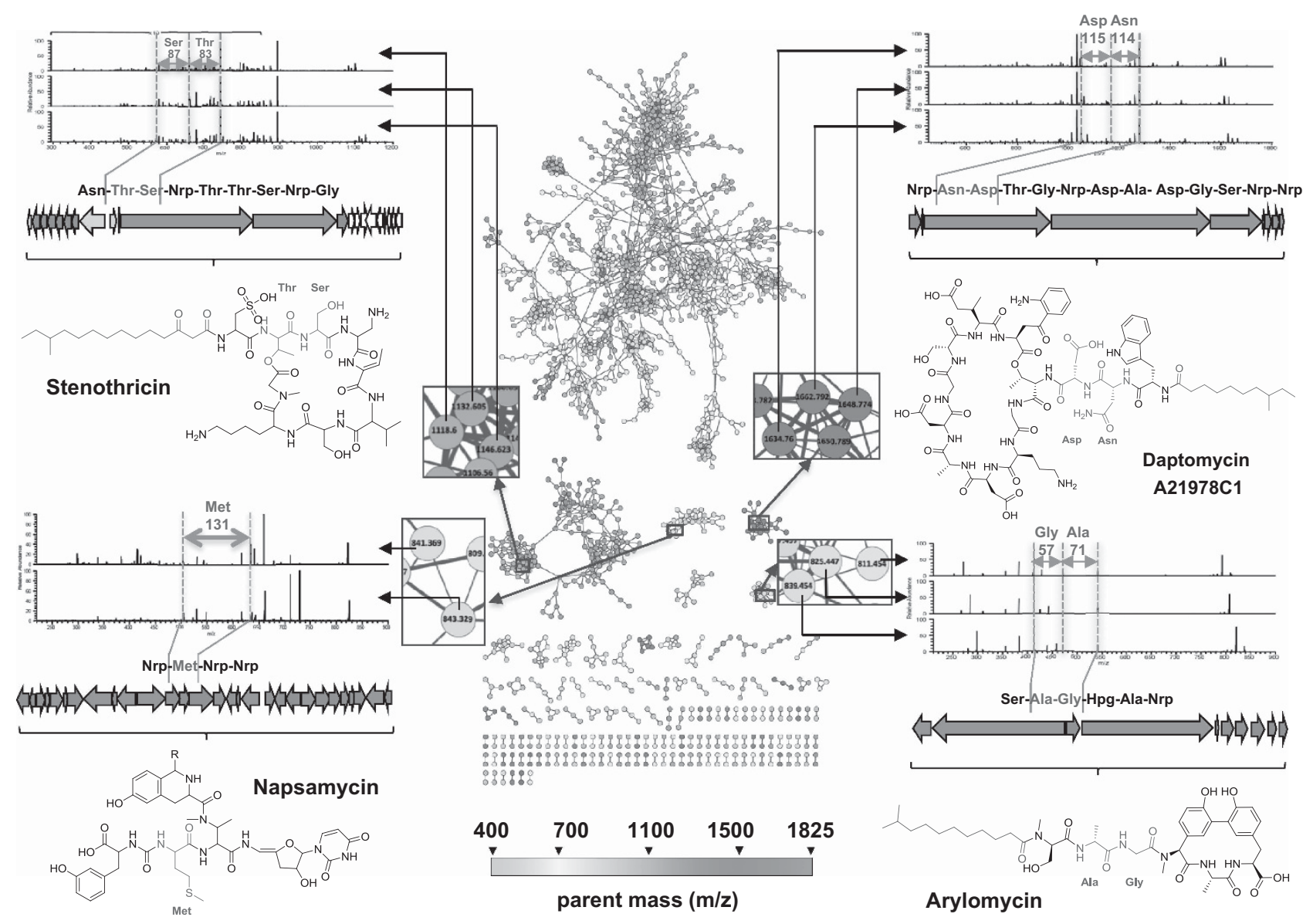

Figure 2 Mapping the molecular network through MS/MS networking and peptidogenomics to reveal the rich metabolic potential of Streptomyces roseosporus. Data sets containing MS/MS spectra collected from S. roseosporus extract were subjected to MS/MS networking analysis and revealed several groups of highly subnetworked ions. Subsequently, analysis of the subnetworks using SST peptidogenomics identified four classes of NRPs products: daptomycin, arylomycin, stenothricin and napsamycin. A full color version of this figure is available at The Journal of Antibiotics journal online.

information we had in the Antimarin database resulted in a candidate molecule, stenothricin, which was discovered in 1974 to be produced by a soil Streptomyces sp. obtained from Zahedan, Iran. ${ }^{28-30}$ Four variants have been reported, three of which were included in Antimarin. ${ }^{28,30}$ Correspondingly, four of the 141 nodes in the stenothricin constellation have $\mathrm{m} / \mathrm{z}$ values of $1118.61,1132.61$, $1146.62,1160.64$ that are within 2 p.p.m. of the calculated mass for these four variants. MS/MS fragmentation of the molecules correlated well with the literature reported stenothricin structures (see Supplementary Notes, and Supplementary Figures S7 and S8).

In addition to the MS/MS data, the gene cluster with 21 predicted genes also agrees with the stenothricin structure (Scheme 1, Supplementary Table S1). Stenothricin contains an $\mathrm{N}$-acyl chain and nine residues; cysteic acid, threonine, serine, 2,3-diaminopropionic acid, dehydrobutyrine, valine, serine, lysine and glycine. The C-terminal glycine is $\mathrm{N}$-methylated and forms a macrocycle involving Thr 2. Lastly, Thr 5 is dehydrated to a dehydrobutyrine. The NRPS assembly-line contains nine NRPS modules on three genes (stenPST). The putative amino acid stereochemical configuration can be inferred by C-domain phylogenetic clustering (see Supplementary Notes and Supplementary Figure S9). ${ }^{31}$ The $N$-methyl group at the C-terminal glycine is in agreement with the methyltransferase domains in the corresponding NRPS modules. Stenothricins contain two unusual amino acids, CysA and Dpr. The genes to make these residues are also present in the gene subnetwork (see Supplementary Notes and Supplementary Figures S10 and S11). Thus, both the MS/MS and the gene cluster match the structure of stenothricin. Finally, NMR data also supported the identification of this group of molecules as being in the stenothricin family of molecules (see Supplementary Notes, and Supplementary Figures S12-S18, Supplementary Table S2). In addition, the large number of nodes in the stenothricin subnetwork revealed a substantial number of analogs with different sized lipid side chains, amino acid substitutions, hydrolysis products and nodes that were $162 \mathrm{Da}$ larger, which is a mass shift often correlated with glycosylation.

Lipopeptides often exhibit promising antimicrobial activities, ${ }^{32}$ such as those exhibited by the well-known anti-infective agents daptomycin and polymyxin. ${ }^{33,34}$ Because of limited quantities of a single purified stenothricin analog ( $\sim 200 \mu \mathrm{g}$ per 600 plates), we used a mixture of stenothricin variants in BioMAP, a screening platform that tests for antimicrobial activity against 15 bacterial strains to generate an activity profile. ${ }^{35}$ On the basis of the cytological profiling as described below, the mixture has the same effect on cells as the singly purified molecule. Contrary to published results where no antimicrobial activity was observed for Bacillus subtilis, ${ }^{29}$ stenothricin is active against both Gram-negative and Gram-positive bacteria including Bacillus subtilis as well as a panel of clinically important pathogenic bacteria such as Staphylococcus epidermis, Enterococcus 

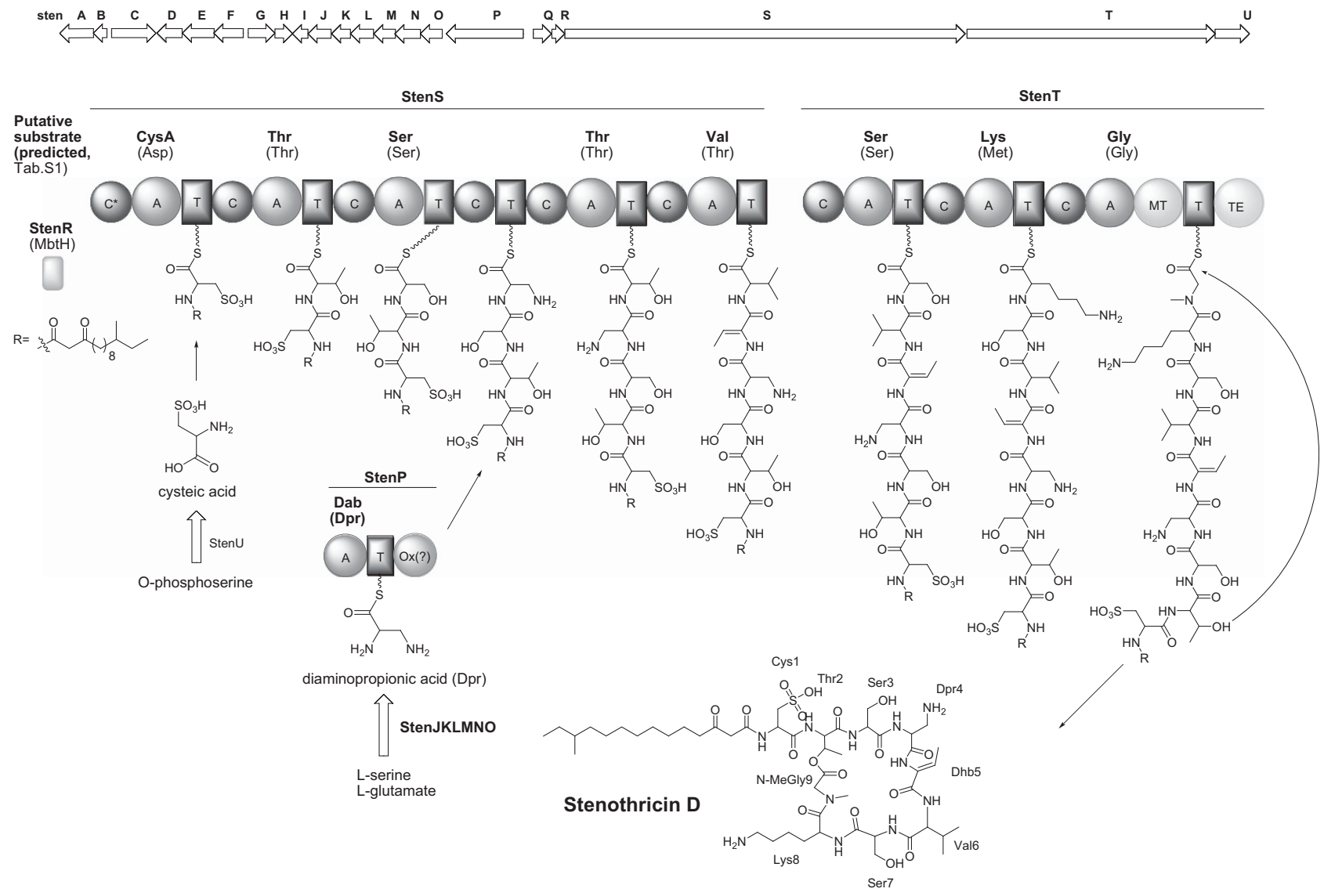

Scheme 1 Proposed biosynthetic pathway of stenothricin. A full color version of this scheme is available at The Journal of Antibiotics journal online.

faecium, Listeria ivanovii, Enterobacter aerogenes and Acinetobacter baumannii with $\mathrm{IC}_{50}$ values ranging from 1 to $27 \mu \mathrm{M}$. The latter are emerging pathogens that cause severe pneumonia, urinary tract infections and septicemia (Supplementary Figure S19). ${ }^{36-38}$ The BioMAP antimicrobial profile ${ }^{16}$ of stenothricin was compared with 72 established profiles of known drugs covering 12 major classes. The results indicated that stenothricin has a distinct activity profile from these 72 known antibiotics, suggesting that it may exhibit a different mode of action compared with the major classes of antibiotics that include cell wall, protein, RNA, and nucleic acid synthesis inhibitors, DNA-damaging agents and additional targets (Figure 3a). ${ }^{35}$

To gain further insight into stenothricin's effect on cells, we employed cytological profiling, ${ }^{39,40}$ a microscopy-based method first utilized in eukaryotic cells, which has recently been applied to bacteria. $^{41}$ Cytological profiling is a modern interpretation of classical pathology and cytology, tools long used for clinical diagnosis and treatment decisions in fields ranging from cancer to infectious disease, with the Gram stain being the most simple and widely employed test to guide treatment of bacterial infections. Briefly, microbial cytological profiling uses high resolution fluorescence microscopy to characterize the subcellular effects of antibiotics on bacterial cells, which respond in specific ways to antibacterial molecules. The subcellular changes induced by antibiotics can be measured using cell stains for the membrane (FM 4-64), DNA (DAPI and SYTOX green) and cell permeability (SYTOX green), much in the same way subcellular changes associated with malignant tumors are visualized using lower resolution methods in the clinical pathology lab. Microbial cytological profiling is particularly useful for assessing the biological activity of membrane active molecules as these activities are difficult to assess using traditional methods. We have used this method together with more traditional methods to pinpoint the mode of action of the SDP toxin as depletion of both components of the proton motive force, which produces a cytological profile that is distinct from forming nonspecific channels (nisin), titration of lipid II precursor for peptidoglycan biogenesis (vancomycin) or from depleting either component of the proton motive force. ${ }^{41}$

We used cytological profiling to compare the activity of stenothricin D, which has the same activity as the stenothricin mixture used in BioMAP (Supplementary Figure S20), to several control compounds using exponentially growing microcultures of B. subtilis. The lowest concentration of stenothricin D to cause an irreversible viability drop was $18 \mu \mathrm{M}$ (Figure 3b), and, after $20 \mathrm{~min}$, the cells showed compact, weakly staining chromosomes and FM 4-64 membrane staining that appeared to fill the cytoplasm with gaps between adjacent cells (Figure 3f). This internal membrane staining phenotype was also observed in E. coli lptD cells (Supplementary Figure S21), demonstrating that it is a conserved response to stenothricin. Treatment with $4.4 \mu \mathrm{M}$ stenothricin D produced a transient growth arrest (Figure $3 \mathrm{~b}$ ). Few cells showed membrane staining filling the cytoplasm and most contained membrane vesicles (Figure $3 \mathrm{~h}$ ) somewhat similar to those seen with vancomycin and triton X-100 treatment (Figures 3j-n). Many stenothricin-treated cells were permeabilized to SYTOX and showed compact chromosomes, unlike vancomycin-treated cells that showed no SYTOX permeability, and triton X-100 or nisin-treated cells that were uniformly 
a

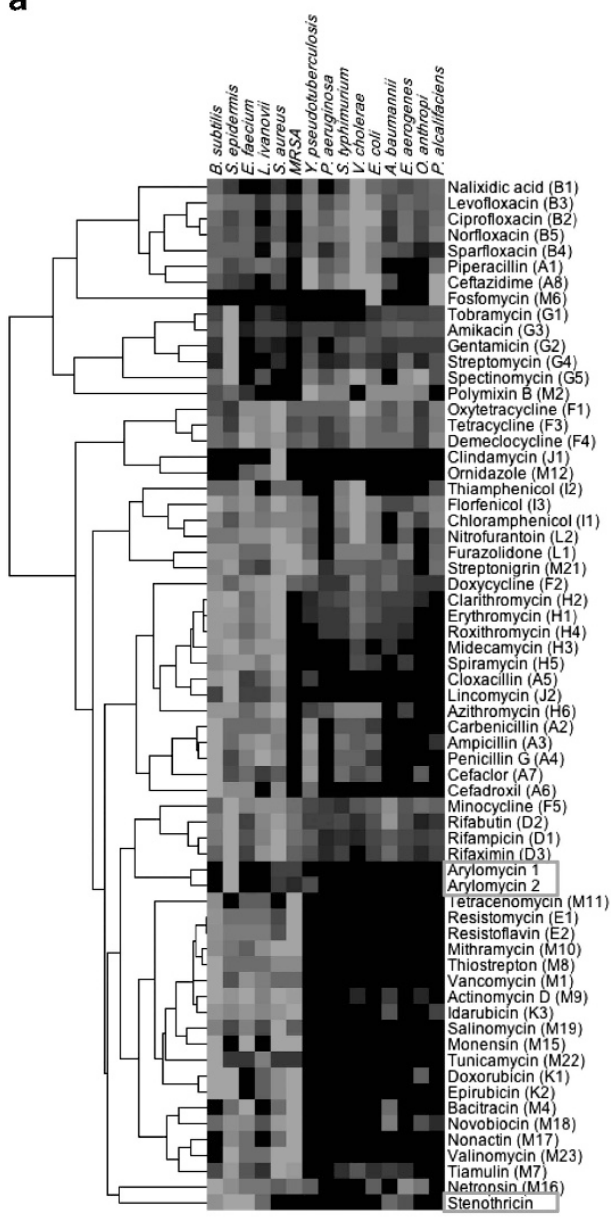

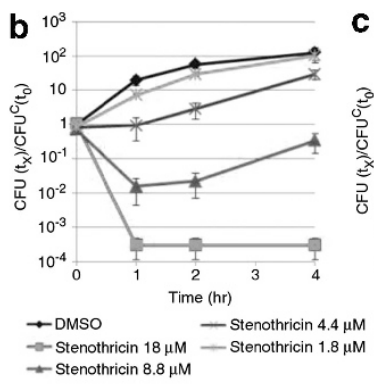

C $10^{3}$

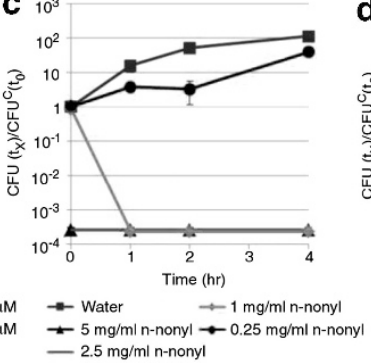

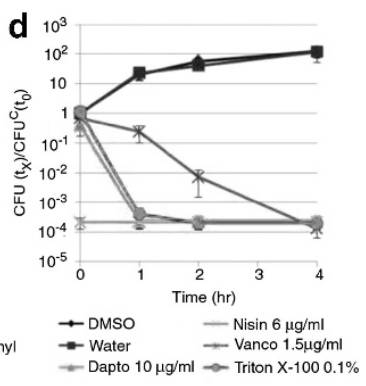

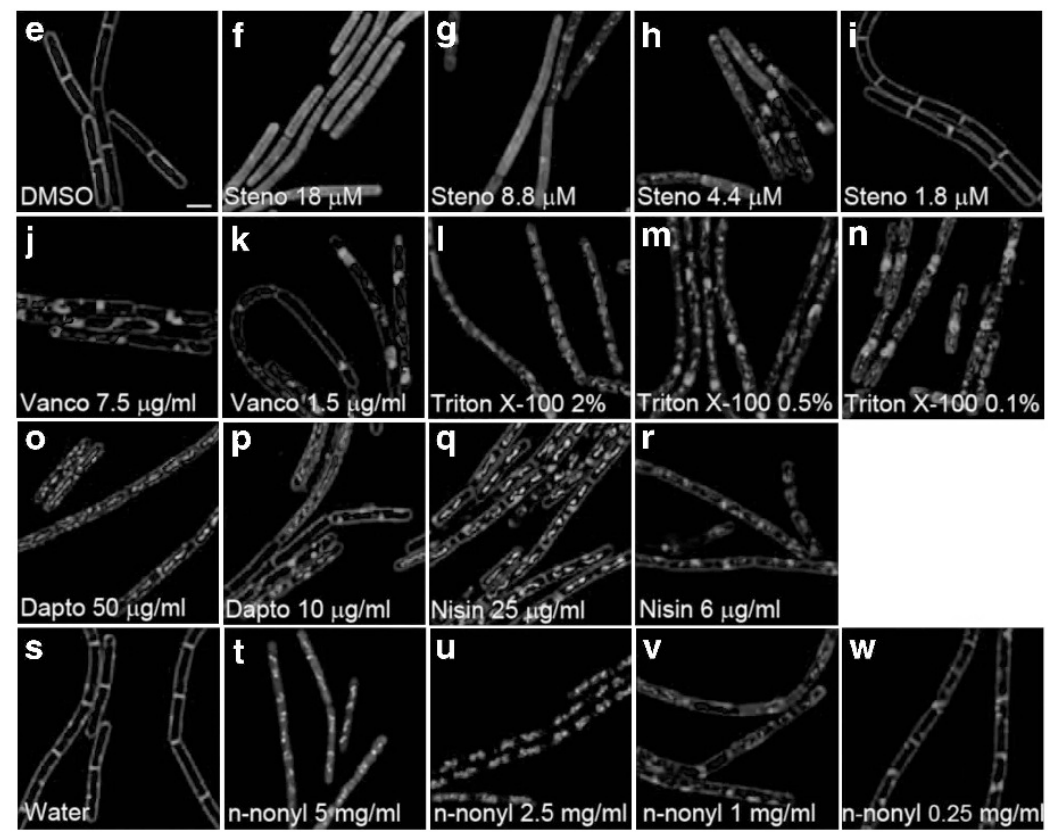

Figure 3 Effects of stenothricin and other antimicrobials on B. subtilis cell architecture and viability. (a) BioMAP profiling compared the antimicrobial activities of stenothricin and arylomycin with 72 known antibiotics covering 12 major classes. Antimicrobial potencies are represented by a red-black color scheme with a gradient from inactive (black) to most potent (red). Antibiotics are alphanumerically labeled as $A$ to $L$ according to structural class. Code $M$ is assigned to antibiotics that are single derivatives without other family members as described in Wong et al. ${ }^{35}$ (b) Effects of different amounts of stenothricin D on PY79 cell viability. (c) Effects of different amounts of $n$-nonyl- $\beta$-D-glucopyranoside on PY79 cell viability. (d) Effects of control treatments on PY79 viability. Cell viability is shown as the ratio of colony-forming units (c.f.u.) at the indicated time and treatment to the cfu at $t_{0}$ for the control (c.f.u. ${ }^{C}$ ). Error bars show the s.e. of $\geqslant 3$ experiments. e-w Fluorescence micrographs of growing PY79 cells treated with (e) $0.5 \%$ DMSO (f) $18 \mu \mathrm{m}$ stenothricin D (g) $8.8 \mu \mathrm{m}$ stenothricin D (h) $4.4 \mu \mathrm{m}$ stenothricin D (i) $1.8 \mu \mathrm{m}$ stenothricin D (j) $7.5 \mu \mathrm{g} \mathrm{ml} \mathrm{m}^{-1}$ vancomycin (k) $1.5 \mu \mathrm{g} \mathrm{ml}-1$ vancomycin (I) $2.0 \%$ Triton X-100 (m) $0.5 \%$ Triton X-100 (n) $0.1 \%$ Triton X-100 (o) $50 \mu \mathrm{g} \mathrm{ml}-1$ daptomycin (p) $10 \mu \mathrm{g} \mathrm{ml}^{-1}$ daptomycin (q) $25 \mu \mathrm{g} \mathrm{ml}-1$ nisin (r) $6 \mu \mathrm{g} \mathrm{ml}-1$ nisin (s) water (t) $5 \mathrm{mg} \mathrm{ml}^{-1} \quad n$-nonyl- $\beta$-D-glucopyranoside (u) $2.5 \mathrm{mg} \mathrm{ml}^{-1} \quad n$-nonyl- $\beta$-D-glucopyranoside (v) $1.0 \mathrm{mg} \mathrm{ml}^{-1} \quad n$-nonyl- $\beta$-D-glucopyranoside (w) $0.25 \mathrm{mg} \mathrm{ml}^{-1} n$-nonyl- $\beta$-D-glucopyranoside for $20 \mathrm{~min}$. Cells are stained with FM 4-64 (red), DAPI (blue) and SYTOX green. SYTOX green is membrane impermeable and only stains cells with compromised membranes. Scale bar represents $2 \mu \mathrm{m}$. A full color version of this figure is available at The Journal of Antibiotics journal online.

permeabilized. Cells treated with $10 \mu \mathrm{g} \mathrm{ml}^{-1}$ of daptomycin were elongated, with small membrane vesicles, and some permeabilization to SYTOX (Figure 3p), appearing distinct from stenothricin D-treated cells. Increasing the amounts of these control compounds to $5-20 \times$ the minimal inhibitory concentration did not produce membrane staining that filled the cytoplasm (Figures $3 \mathrm{j}, 1$, o and $\mathrm{q}$ ). Thus, stenothricin D-treated cells appear distinct from all other compounds we have tested thus far.

Membrane staining that fills the cytoplasm might be expected for a molecule with detergent like properties, although triton X-100 did not produce this cytological profile. We therefore sought a detergent more similar in structure to stenothricin D than triton X-100. We chose to test $n$-nonyl- $\beta$-D-glucopyranoside, a non-ionic detergent with an alkyl chain of similar length to stenothricin $D$, but with the depsipeptide moiety of stenothricin replaced with a glycoside. When B. subtilis cells were treated with this detergent at $5 \mathrm{mg} \mathrm{ml}^{-1}$, we observed a similar membrane staining as seen with stenothricin D-treated cells (Figures $3 t$ and $\mathrm{u}$ ), although the chromosomes stained more brightly with SYTOX than did stenothricin-treated cells. Treatment with $1 \mathrm{mg} \mathrm{ml}^{-1} n$-nonyl- $\beta$-D-glucopyranoside (the minimal inhibitory concentration) resulted in an intermediate phenotype where some cells had minimal cytoplasmic staining (Figure 3v). Overall, the cytological profile of $n$-nonyl- $\beta$-D-glucopyranoside-treated cells is similar, but not identical to that of stenothricin D-treated cells. Thus, cytological profiling suggests that, at least at high concentrations, stenothricin D acts in a manner similar to certain detergents. The BioMAP profiling does not utilize any detergents, and thus would not cluster stenothricin with any compounds.

The above results show that $S$. roseosporus produced a large panel of NRPS-derived antibiotics, including previously unreported analogs of 
known molecules involving truncations, glycosylations, hydrolysis products and biosynthetic intermediates and/or shunt products and that all can be visualized using MS/MS networking (Supplementary Figure S3). Specifically, MS/MS networking highlights that S. roseosporus produces at least four NRPS-derived molecular families with different mode of actions. Daptomycin acts by disrupting membrane structure, ${ }^{42,43}$ arylomycin as a type II signal peptidase inhibitor, ${ }^{13,44}$ napsamycin as a bacterial translocase I inhibitor, ${ }^{25}$ and stenothricin disrupts the membrane in a manner that is distinct from daptomycin but somewhat similar to $n$-nonyl- $\beta$-D-glucopyranoside. The diversity of antibacterial compounds produced by $S$. roseosporus highlights the importance of developing new approaches to characterize the 'molecular network' of an organism and thus more deeply interrogate the biosynthetic capacities of microorganisms, which is the goal to facilitate the mining of high-value molecules of biotechnological importance that originate from natural sources.

\section{METHODS}

Materials, detailed experimental procedures, Supplementary texts and Figures are described in Supplementary Information.

\section{ACKNOWLEDGEMENTS}

Financial support was provided by the National Institute of Health (GM086283 to PCD; AI095125 to PCD and KP; GM097509 to PD and BSM; TW006634 to RGL; CA100851 to WHG). WTL was supported, in part, by a study abroad grant (SAS-98116-2-US-108) from Taiwan. WRW was supported by the Malaysian Biotechnology Corporation QB3-Malaysia program.

1 Newman, D. J., Cragg, G. M. \& Snader, K. M. Natural products as sources of new drugs over the period 1981-2002. J. Nat. Prod. 66, 1022-1037 (2003).

2 Demain, A. L. Antibiotics: natural products essential to human health. Med. Res. Rev. 29, 821-842 (2009)

3 Newman, D. J. \& Cragg, G. M. Natural products as sources of new drugs over the last 25 years. J. Nat. Prod. 70, 461-477 (2007).

4 Klevens, R. M. et al. Invasive methicillin-resistant Staphylococcus aureus infections in the United States. JAMA 298, 1763-1771 (2007)

5 Chambers, H. F. \& Deleo, F. R. Waves of resistance: Staphylococcus aureus in the antibiotic era. Nat. Rev. Microbiol. 7, 629-641 (2009).

6 Fischbach, M. A. \& Walsh, C. T. Antibiotics for emerging pathogens. Science 325, 1089-1093 (2009).

7 Lewis, K. Antibiotics: Recover the lost art of drug discovery. Nature 485, 439-440 (2012).

8 Li, J. W. \& Vederas, J. C. Drug discovery and natural products: end of an era or an endless frontier? Science 325, 161-165 (2009).

9 Walsh, C. Where will new antibiotics come from? Nat. Rev. Microbiol. 1, 65-70 (2003).

10 Liu, W. T. et al. Interpretation of Tandem Mass Spectra Obtained from Cyclic Nonribosomal Peptides. Anal. Chem. 81, 4200-4209 (2009).

$11 \mathrm{Ng}$, J. et al. Dereplication and de novo sequencing of nonribosomal peptides. Nat. Methods 6, 596-U565 (2009)

12 Kersten, R. D. et al. A mass spectrometry-guided genome mining approach for natural product peptidogenomics. Nat. Chem. Biol. 7, 794-802 (2011)

13 Liu, W. T., Kersten, R. D., Yang, Y. L., Moore, B. S. \& Dorrestein, P. C. Imaging mass spectrometry and genome mining via short sequence tagging identified the antiinfective agent arylomycin in Streptomyces roseosporus. J. Am. Chem. Soc. 133, 18010-18013 (2011).

14 Watrous, J. et al. Mass spectral molecular networking of living microbial colonies. Proc. Natl Acad. Sci. USA 109, E1743-E1752 (2012).

15 Yang, Y. L., Xu, Y., Straight, P. \& Dorrestein, P. C. Translating metabolic exchange with imaging mass spectrometry. Nat. Chem. Biol. 5, 885-887 (2009).
16 Aebersold, R. \& Mann, M. Mass spectrometry-based proteomics. Nature 422, 198-207 (2003)

17 Patti, G. J., Yanes, O. \& Siuzdak, G. Innovation: metabolomics: the apogee of the omics trilogy. Nat. Rev. Mol. Cell. Biol. 13, 263-269 (2012).

18 Shannon, P. et al. Cytoscape: A software environment for integrated models of biomolecular interaction networks. Genome Res. 13, 2498-2504 (2003).

19 Smoot, M. E., Ono, K., Ruscheinski, J., Wang, P. L. \& Ideker, T. Cytoscape 2.8: new features for data integration and network visualization. Bioinformatics 27, 431-432 (2011).

20 Debono, $M$. et al. A21978C a complex of new acidic peptide antibiotics: isolation, chemistry, and mass spectral structure elucidation. J. Antibiot. 40, 761-777 (1987).

21 Baltz, R. H. Biosynthesis and genetic engineering of lipopeptide antibiotics related to daptomycin. Curr. Top. Med. Chem. 8, 618-638 (2008).

22 Miao, V. et al. Daptomycin biosynthesis in Streptomyces roseosporus: cloning and analysis of the gene sub-network and revision of peptide stereochemistry. Microbiology 151, 1507-1523 (2005).

23 Holtzel, A et al. Arylomycins A and B, new biaryl-bridged lipopeptide antibiotics produced by Streptomyces sp. Tu 6075. II. Structure elucidation. J. Antibiot. 55, 571-577 (2002)

24 Jin, X. et al. Biosynthesis of new lipopentapeptides by an engineered strain of Streptomyces sp. Biotechnol. Lett. 34, 2283-2289 (2012).

25 Kaysser, L. et al. Identification of a napsamycin biosynthesis gene sub-network by genome mining. Chembiochem. 12, 477-487 (2011).

26 Medema, M. H. et al. antiSMASH: rapid identification, annotation and analysis of secondary metabolite biosynthesis gene sub-networks in bacterial and fungal genome sequences. Nucleic Acids Res. 39, W339-W346 (2011).

27 Stachelhaus, T., Mootz, H. D. \& Marahiel, M. A. The specificity-conferring code of adenylation domains in nonribosomal peptide synthetases. Chem. Biol. 6, 493-505 (1999).

28 Konig, W. A., Engelfried, C., Hagenmaier, H. \& Kneifel, H. Structure of the peptide antibiotic Stenothricin. Justus. Liebigs. Ann. Chem. 1976, 2011-2020 (1976).

29 Hasenbohler, A., Kneifel, H., Konig, W. A., Zahner, H. \& Zeiler, H. J. Stenothricin, a new inhibitor of the bacterial cell wall synthesis. Arch. Microbiol. 99, 307-321 (1974)

30 Rinken, M., Lehmann, W. D. \& Konig, W. A. The structure of Stenothricin - revision of a previous structure assignment. Liebigs Ann. Chem. 1672-1684 (1984).

31 Rausch, C., Hoof, I., Weber, T., Wohlleben, W. \& Huson, D. H. Phylogenetic analysis of condensation domains in NRPS sheds light on their functional evolution. BMC. Evol. Biol. 7, 78-92 (2007)

32 Pirri, G., Giuliani, A., Nicoletto, S. F., Pizzuto, L. \& Rinaldi, A. C. Lipopeptides as antiinfectives: a practical perspective. Cent. Eur. J. Biol. 4, 258-273 (2009).

33 Baltz, R. H., Miao, V. \& Wrigley, S. K. Natural products to drugs: daptomycin and related lipopeptide antibiotics. Nat. Prod. Rep. 22, 717-741 (2005).

34 Martin, N. I. et al. Isolation, structural characterization, and properties of mattacin (polymyxin M), a cyclic peptide antibiotic produced by Paenibacillus kobensis M. J. Biol. Chem. 278, 13124-13132 (2003).

35 Wong, W. R., Oliver, A. G. \& Linington, R. Development of antibiotic mode of action profile screening for the classification and discovery of natural product antibiotics. Chem. Biol. 19, 1483-1495 (2012).

36 Peleg, A. Y., Seifert, H. \& Paterson, D. L. Acinetobacter baumannii: emergence of a successful pathogen. Clin. Microbiol. Rev. 21, 538-582 (2008).

37 Maragakis, L. L. \& Perl, T. M. Acinetobacter baumannii: epidemiology, antimicrobial resistance, and treatment options. Clin. Infect. Dis. 46, 1254-1263 (2008).

38 Dijkshoorn, L., Nemec, A. \& Seifert, H. An increasing threat in hospitals: multidrugresistant Acinetobacter baumannii. Nat. Rev. Microbiol. 5, 939-951 (2007).

39 Lorang, J. \& King, R. W. Cytological profiling: providing more haystacks for chemists' needles. Genome. Biol. 6, 228 (2005).

40 Perlman, Z. E. et al. Multidimensional drug profiling by automated microscopy. Science 306, 1194-1198 (2004).

41 Lamsa, A., Liu, W. T., Dorrestein, P. C. \& Pogliano, K. The Bacillus subtilis cannibalism toxin SDP collapses the proton motive force and induces autolysis. Mol. Microbiol. 84, 486-500 (2012).

42 Steenbergen, J. N., Alder, J., Thorne, G. M. \& Tally, F. P. Daptomycin: a lipopeptide antibiotic for the treatment of serious Gram-positive infections. J. Antimicrob. Chemother. 55, 283-288 (2005)

43 Pogliano, J., Pogliano, N. \& Silverman, J. A. Daptomycin-mediated reorganization of membrane architecture causes mislocalization of essential cell division proteins. J. Bacteriol. 194, 4494-4504 (2012).

44 Paetzel, M., Goodall, J. J., Kania, M., Dalbey, R. E. \& Page, M. G. Crystallographic and biophysical analysis of a bacterial signal peptidase in complex with a lipopeptide-based inhibitor. J. Biol. Chem. 279, 30781-30790 (2004).

Supplementary Information accompanies the paper on The Journal of Antibiotics website (http://www.nature.com/ja) 